\title{
Asymmetric Chiral Metamaterial Superstrate for Patch Antenna Polarization Transformation
}

\author{
Oscar Fernández, Álvaro Gómez, José Basterrechea, Angel Vegas \\ Dpto. de Ingeniería de Comunicaciones, Universidad de Cantabria, Santander, Spain. \\ oscar.fernandez@unican.es, alvaro.gomez@unican.es, jose.basterrechea@unican.es, angel.vegas@unican.es.
}

\begin{abstract}
Planar superstrates constituted by Chiral Metamaterials structures can transform the radiation properties of an antenna. In this communication, an asymmetric chiral metamaterial based structure is proposed. Due to the lack of $\mathrm{C}_{4}$ symmetry in the design, the transmission coefficients depend on the incident wave polarization and display high transmission for one circular polarization but low transmission for the other one.

The proposed structure is then used to improve the radiation characteristics of a microstrip patch antenna. Taking advantage of the properties of the asymmetric chiral structure, the polarization of the radiated field is modified, transforming the linear polarization of the microstrip antenna into a circular one.
\end{abstract}

Index Terms-Chiral metamaterial, patch antenna, superstrate, circular polarization.

\section{INTRODUCTION}

Metamaterials are artificial composite materials that exhibit properties neither available in the nature nor observed in their constituents, or enhance the composite features relative to the individual properties of its constituents. Among them, we highlight the Chiral Metamaterials (CMM), which present high polarization rotation angles and can exhibit negative refractive index [1].

In the antenna design, chiral metamaterials have been applied to create superstrates [2]-[6]. A correctly designed CMM superstrate can transform the radiation properties of an antenna, increasing its gain, reducing the half power beam width (HPBW) and/or modifying the polarization of the radiated field.

Several papers [2]-[4], use a superstrate in order to increase the gain of circularly polarized patch antennas. However, only a few papers [5], [6] are oriented to modify the polarization of its radiated field. In a previous work [5], the authors presented a CMM superstrate placed over a linear polarized patch antenna which increased the antenna gain for one of the circularly polarized waves (left handed - LHCP or right handed - RHCP). The resulting radiated electric field was elliptically polarized.

In this paper the authors present a new superstrate, constituted by a novel asymmetric chiral metamaterial structure, which can be used as a complement of a patch antenna in W-band (75-110 GHz). The proposed CMM superstrate is designed to transform the linear polarized field

This work has been partially supported by the Spanish Government MINECO through the FEDER co-funded Research Project TEC2014-55463C3-3-P and the Research Project TEC2012-33321. radiated by the patch antenna into a circular polarized one: the superstrate filters out one of the circularly polarized waves and transmits the other with high gain. Therefore, the placement of the superstrate above a simple antenna provides an equivalent antenna with radiation characteristics found on more complex antennas.

The combination of the aforementioned patch antenna and the asymmetric CMM superstrate, that we call ACMM based antenna, can be used for applications in the lower part of the Terahertz band where directive antennas with circular polarization are needed, e.g. imaging applications [7], [8].

\section{ANTENNA WITH ACMM SUPERSTRATE}

In this section the radiation features of the ACMM antenna are numerically analyzed with the finite differences time domain and the finite elements method in the frequency domain engines of EMPro 3D electromagnetic simulator of Keysight Technologies. With this idea in mind, the geometry of the patch antenna is firstly introduced. Secondly, the ACMM superstrate is characterized. Finally, the radiation properties of the whole ACMM based antenna are presented.

\section{A. Patch Antenna}

The radiant element of the ACMM antenna is a patch antenna (Fig. 1) excited by slot which is constituted by two layers [5]. The bottom layer uses Rogers ULTRALAM 2000 substrate with a dielectric thickness of 4 mils $(0.102 \mathrm{~mm}), \varepsilon_{\mathrm{r}}=$ $2.5, \operatorname{tg} \delta=0.0019$ and a copper thickness of $18 \mu \mathrm{m}$. This layer also contains a $100 \Omega$ microstrip feed line $(3.7 \times 0.09 \mathrm{~mm})$ located at its rear face and the ground plane where the slot $(0.7$ $\times 0.09 \mathrm{~mm}$ ) is settled at the top face of the same layer. The top layer is the radiant substrate, air in this case, with a thickness of 5 mils. Over this layer it is placed the metallic rectangular patch $(1 \times 1.1 \mathrm{~mm})$. The proposed antenna is designed to operate at a central frequency of $100 \mathrm{GHz}$ with a bandwidth of $5 \mathrm{GHz}$.

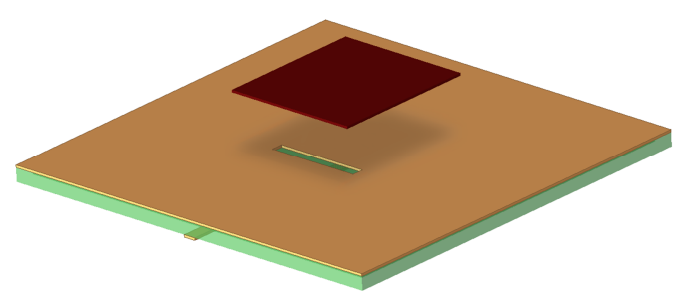

Fig. 1. Slot coupled microstrip patch antenna. 


\section{B. ACMM Superstrate}

The asymmetric CMM structure, presented by the authors in [9], is designed to transform a linear polarized wave into a circular polarized one. The geometrical dimensions of the unit cell (Fig. 2) are given by $l_{1}=0.430 \mathrm{~mm}, l_{2}=0.7 \mathrm{~mm}, w=40$ $\mu \mathrm{m}, g_{1}=0.6 \mathrm{~mm}$ and $g_{2}=0.86 \mathrm{~mm}$. The substrate has a thickness of $100 \mu \mathrm{m}$ with a dielectric relative constant of $\varepsilon_{\mathrm{r}}=$ 2.4 and $\operatorname{tg} \delta=0.0001$. The metallization thickness is $1 \mu \mathrm{m}$.
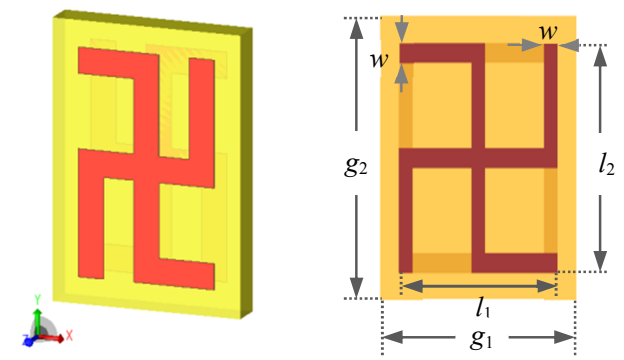

Fig. 2. Asymmetric conjugated gammadion CMM unit cell

Due to the lack of $\mathrm{C}_{4}$ symmetry of the CMM structure, its linear transmission coefficients depend on the incident wave polarization orientation (See Fig. 3). As a consequence, the design produces a huge difference between the co-polar transmission coefficients $t_{\mathrm{xx}}$ and $t_{\mathrm{yy}}$.
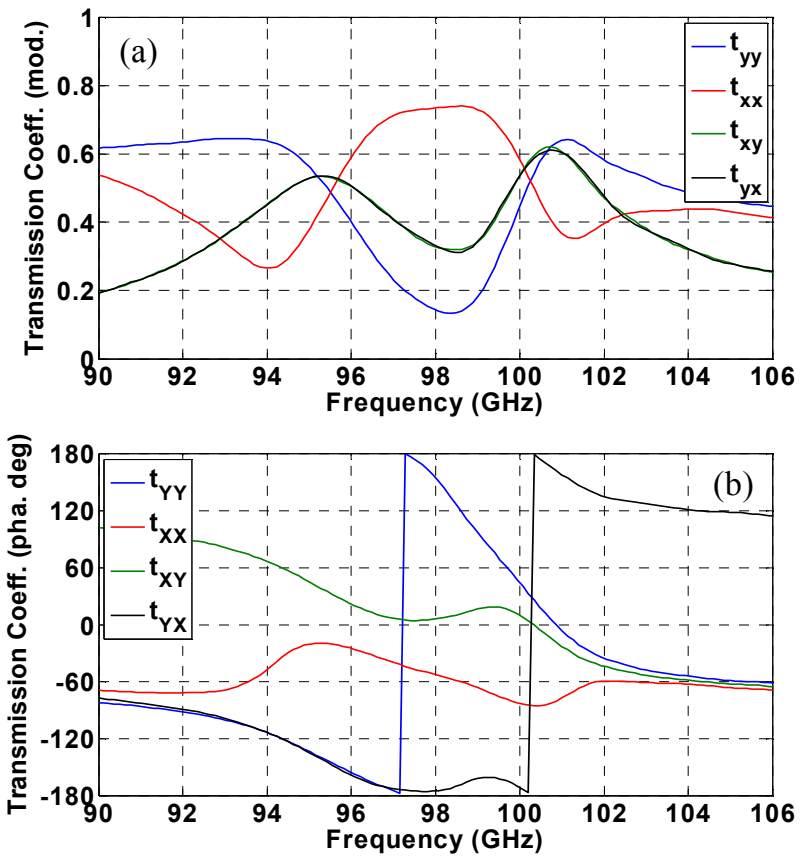

Fig. 3. Linear transmission coeff. (a) module and (b) phase

The linear to circular transmission matrix, $\boldsymbol{T}_{c l}[10]$, relates the circularly polarized transmitted fields, $E_{+}^{t}$ and $E_{-}^{t}$, with the linear polarized field that impinges on the chiral metamaterial slab, $E_{x}^{i}$ and $E_{y}^{i}$.

$$
\begin{aligned}
\left(\begin{array}{c}
E_{+}^{t} \\
E_{-}^{t}
\end{array}\right) & =\left(\begin{array}{c}
E_{x}^{t}+j E_{y}^{t} \\
E_{x}^{t}-j E_{y}^{t}
\end{array}\right)=\frac{1}{\sqrt{2}}\left(\begin{array}{ll}
t_{x x}+j t_{y x} & t_{x y}+j t_{y y} \\
t_{x x}-j t_{y x} & t_{x y}-j t_{y y}
\end{array}\right)\left(\begin{array}{c}
E_{x}^{i} \\
E_{y}^{i}
\end{array}\right) \\
& =\left(\begin{array}{ll}
t_{+x} & t_{+y} \\
t_{-x} & t_{-y}
\end{array}\right)\left(\begin{array}{l}
E_{x}^{i} \\
E_{y}^{i}
\end{array}\right)=\boldsymbol{T}_{c l}\left(\begin{array}{c}
E_{x}^{i} \\
E_{y}^{i}
\end{array}\right)
\end{aligned}
$$

being $t_{+\mathrm{x}}\left(t_{-\mathrm{y}}\right)$ the transmission coefficient of a RHCP (LHCP) wave generated by an incident field linearly polarized parallel to the $x$-axis ( $y$-axis).

From the numerical simulations of this ACMM structure (Fig. 3), we obtain that at $100.1 \mathrm{GHz} t_{y x}=t_{x x} \cdot e^{-j \pi / 2}=$ $t \cdot e^{-j \pi / 2}$, and $|t|=0.56$. With these values, the transmission coefficients $t_{+_{x}}$ and $t_{-x}$ can be expressed as (2). This means that when the incident field is $E_{x}$, the ACMM only transmits one of the circularly polarized components with a transmission coefficient of $\sqrt{2} t$. This result is confirmed with the simulation results presented in Fig. 4.

$$
\left(\begin{array}{l}
t_{+x} \\
t_{-x}
\end{array}\right)=\frac{1}{\sqrt{2}}\left(\begin{array}{l}
t+j t e^{-j \pi / 2} \\
t-j t e^{-j \pi / 2}
\end{array}\right)=\sqrt{2}\left(\begin{array}{l}
t \\
0
\end{array}\right)
$$

As a result, the structure acts as a circular polarizer, transforming a linear polarized incident wave parallel to the shorter side of the ACMM structure, $E_{x}$ in the case of Fig. 2, into a circularly polarized transmitted one $E_{+}^{t}$.

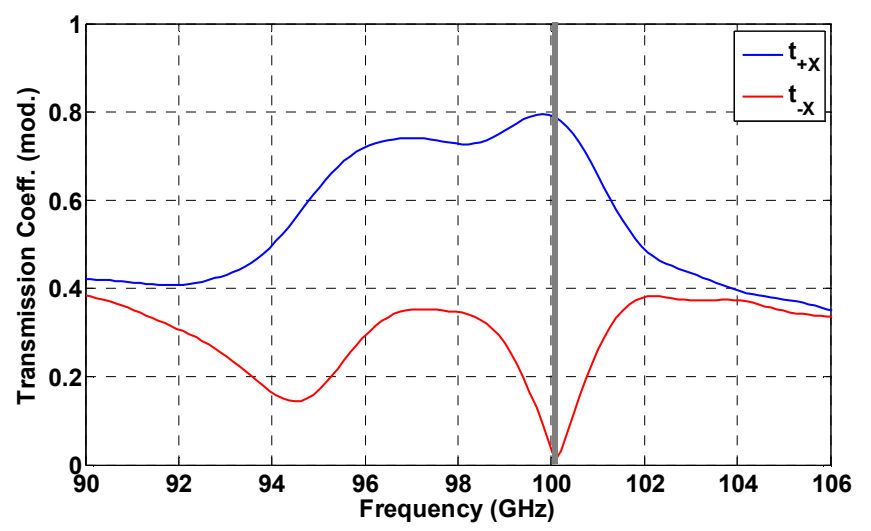

Fig. 4. Linear to circular transmission coeff. for a $x$-polarized incident field

\section{ACMM based Antenna}

Finally, the patch antenna and the asymmetric CMM described in previous sections are combined to form the ACMM based antenna (Fig. 5). The antenna and the superstrate are parallel and aligned in such a way that the linearly polarized radiated field (following the $y$-axis in the example of Fig. 5) is parallel to the short side of the ACMM structure unit cell.

The role of the superstrate is to exploit the filtering properties provided by the ACMM structure over one of the circular components. Thus the linear polarization radiated by the patch antenna becomes circularly polarized. 


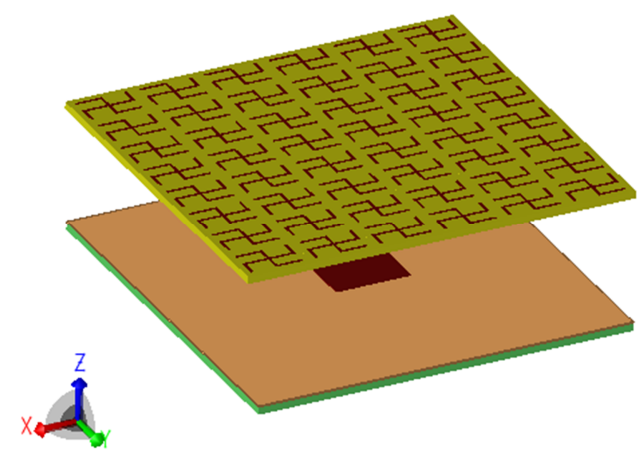

Fig. 5. Microstrip patch antenna with the ACMM based superstrate

The effect of the superstrate is studied as a function of the separation $d$ between the patch and the superstrate. This parameter affects not only the gain of the antenna, but also the polarization.

The first studied effect has been the influence of the superstrate on the antenna gain. Fig. 6 presents the total gain $\mathrm{G}$ of the ACMM based antenna and the gain of its circular right handed $\left(\mathrm{G}_{+}\right)$and left handed $\left(\mathrm{G}_{-}\right)$components as a function of its separation with respect to the patch. The maximum value of $\mathrm{G}$ is $14.2 \mathrm{dBi}$ and it is obtained at $d=1.6$ $\mathrm{mm}$. This represents an increase of $6 \mathrm{~dB}$ with respect to the gain of the single patch antenna. Comparing the electric field distribution in the H-plane of both the single patch antenna and the ACMM based antenna (Fig. 7) we can check that the superstrate concentrates the field in the direction perpendicular to the superstrate.

Moreover, focusing our attention on the CP components gain, the inclusion of the superstrate improves the transmission of the RH component while minimizes the LH one. For low separations, $d<1.5 \mathrm{~mm}$, the gains of both components are similar; this means that there is no polarization conversion. For separations greater than $1.5 \mathrm{~mm}$ both components present a very different behavior.

In the case of the RH component, $\mathrm{G}_{+}$presents its higher values, greater than $12 \mathrm{dBi}$, for separations in the range of 1.5 and $1.9 \mathrm{~mm}$ with a maximum of $13.9 \mathrm{dBi}$ for $d=1.6 \mathrm{~mm}$ (Fig. $6)$.

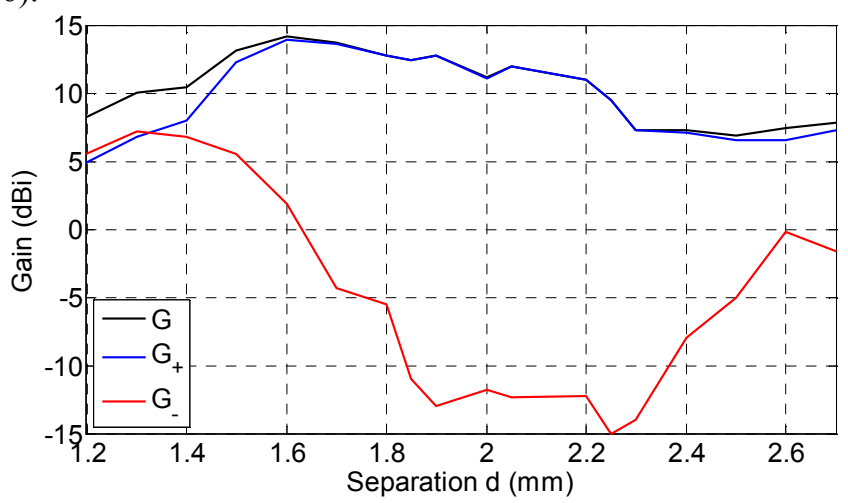

Fig. 6. Total gain $\mathrm{G}, \mathrm{G}_{+}$and $\mathrm{G}_{\text {. of }}$ the ACMM antenna
The radiation pattern also depends on the parameter $d$. Fig. 8 shows the radiation pattern of $\mathrm{G}_{+}$and $\mathrm{G}_{-}$in the H-plane for four different values of $d$. It can be seen that for $d=1.4 \mathrm{~mm}$, the radiation pattern resembles a simple patch antenna pattern, but with a narrower half power beamwidth (HPBW) of $48^{\circ}$. For $\mathrm{d}=1.7 \mathrm{~mm}$ and $2 \mathrm{~mm}$ the $3 \mathrm{~dB}$ beamwidth of the main lobe is reduced to a minimum, $26^{\circ}$, nearly a third of the single patch antenna HPBW $\left(76^{\circ}\right)$. In these cases secondary lobes appear at $\pm 90^{\circ}$ from the boresight direction due to the gap between the cover and the antenna ground plane. For $d \geq 2$ $\mathrm{mm}$ it also appears side lobes at $\pm 40^{\circ}$ from the boresight direction that reduce the gain. The greater the distance $d$, the smaller is the sidelobe level, being nearly zero for $d=2.5$ $\mathrm{mm}$.
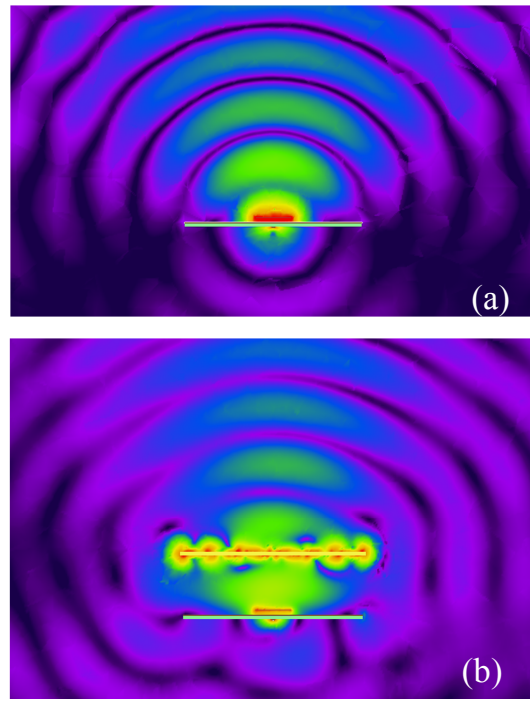

Fig. 7. E-field distribution in H-plane of a) patch antenna b) ACMM antenna with $d=1.7 \mathrm{~mm}$
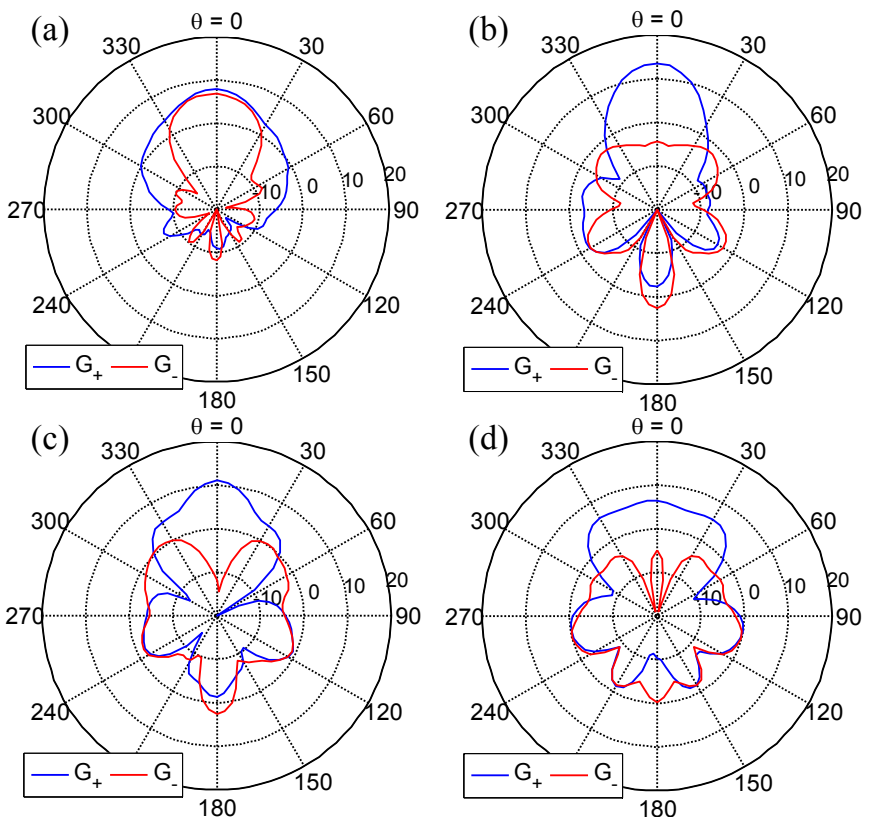

Fig. 8. Radiation pattern in H-plane of the ACMM based antenna with a) $d=1.4 \mathrm{~mm}$, b) $d=1.7 \mathrm{~mm}$, c) $d=2.0 \mathrm{~mm}$ and d) $d=2.5 \mathrm{~mm}$ 
Meanwhile the LH component, in contrast with the $\mathrm{RH}$ component, presents low gain values in the boresight direction of the antenna. As it can be seen in Fig. 6, the gain decreases sharply in the range from 1.5 and $1.9 \mathrm{~mm}$. From this value to $2.3 \mathrm{~mm}$, the LH component is filtered out due to the low values of $\mathrm{G}_{-}$. Fig. 8 shows that for the separations 1.7, 2 and $2.5 \mathrm{~mm}$, the radiation pattern presents, in general, gain values lower than $0 \mathrm{dBi}$. In these cases, the main lobe is in the backward direction.

These great differences between the two circular components are reflected on the polarization of the radiated wave. The resulting polarization type will be characterized through the axial ratio (AR) [11], i.e., the ratio of the major axis to the minor axis of the polarization ellipse. Values of AR less than $3 \mathrm{~dB}$ represent circular polarization whereas for higher values polarization is elliptical.

Fig. 9 represents the axial ratio of the ACMM based antenna as a function of $d$ in the boresight direction. For small separations, lower that $1.7 \mathrm{~mm}$, the $\mathrm{AR}$ is greater than $3 \mathrm{~dB}$ therefore the polarization is elliptical. In the range of 1.7 and $2.4 \mathrm{~mm}$, the ACMM antenna radiates a circularly polarized wave with $\mathrm{AR}<3 \mathrm{~dB}$. Within these values of $d$ the superstrate is far enough from the patch to behave as it was predicted. The best separation, in terms of AR, is $d=1.9 \mathrm{~mm}$ where a minimum value of $\mathrm{AR}, 0.8 \mathrm{~dB}$, is obtained. In this case, the $\mathrm{AR}$ beamwidth is $36^{\circ}, 10$ degrees greater than the $3 \mathrm{~dB}$ beamwidth.

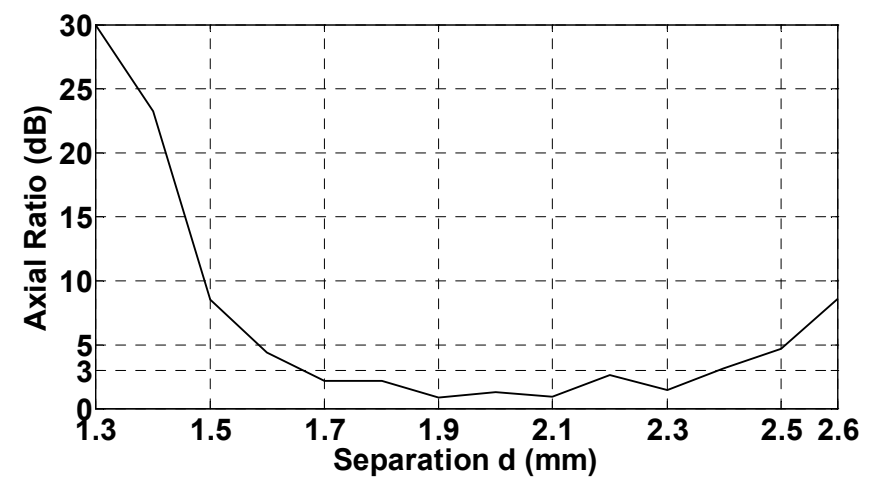

Fig. 9. Axial ratio of the ACMM based antenna as a function of the parameter $d$.

\section{CONCLUSIONS}

In this work an antenna designed with the aim of obtaining high gain and circular polarization from a simple antenna using a chiral metamaterial cover is presented. This antenna is formed by the combination of a linearly polarized single microstrip patch with a superstrate constituted by a novel asymmetric chiral metamaterial structure. This chiral metamaterial transforms the linearly polarized incident wave into a circularly polarized transmitted one.

The results show a strong dependence on the antenna performance with the separation of the superstrate to the patch (d). For small gaps, $d<1.7 \mathrm{~mm}$, the antenna can provide high gain but with an elliptically polarized field. For separations greater than $2 \mathrm{~mm}$, the antenna exhibits low gain. The optimum separations occur between 1.7 and $2 \mathrm{~mm}$, wherein high gain (up to $13.6 \mathrm{dBi}$ ), reduced HPBW and circular polarization is obtained.

\section{REFERENCES}

[1] J. B. Pendry, "A chiral route to negative refraction," Science, vol. 306, no. 5700, pp. 1353-1355, Nov 2004.

[2] M. Malathong, A. Sonsilphong, W. Panpradit and N. Wongkasem, "Chiral Metamaterial Based circularly polarized microstrip antennas", Antennas and Propagation in Wireless Communications (APWC), 2011 IEEE-APS Topical Conference on, pp. 898-901, Torino, Sep. 12-16, 2011.

[3] D. Zarifi, H. Oraizi, and M. Soleimani, "Improved performance of circularly polarized antenna using semi-planar chiral metamaterial covers", Progress In Electromagnetics Research Proceedings, pp. 337354, Moscow, Aug. 19-23, 2012.

[4] D. K. Rongas, S. A. Amanatiadis, A. I. Dimitriadis and N. V. Kantartzis, "Directivity-Enhanced Log-Spiral Antenna through a Chiral Metamaterial Superstrate", $7^{\text {th }}$ European Conference on Antennas and propagation (EuCAP), pp. 2079-2083, Gothenburg, Apr. 8-12, 2013.

[5] J. Gutiérrez, O. Fernández, J.P. Pascual, Á. Gómez, A. Tazón, A. Vegas and Á. Mediavilla, "W-Band Linear Array of Planar Antennas and Chiral Metamaterial Cover", International Journal of Microwave and Optical Technology., vol. 9, no. 6, pp. 384-393. Nov. 2014.

[6] Y. Liu, Y. Qi, K. Song, S. Gu, and X. Zhao, "Circularly polarized patch antenna based on chiral metamaterial", Progress In Electromagnetics Research Symposium Proceedings, pp. 1025-1027, Taipei, Mar. 25-28, 2013.

[7] S. Oka, H. Togo, N. Kukutsu and T. Nagatsuma, "Latest trends in millimeter-wave imaging technology", Progress In Electromagnetics Research Letters, vol. 1, pp. 197-204, 2008.

[8] R. Appleby and H. B. Wallace, "Standoff detection of weapons and contraband in the $100 \mathrm{GHz}$ to $1 \mathrm{THz}$ region", IEEE Transactions on Antennas and Propagation., vol. 55, no. 11, pp.2944-2956, Nov. 2007.

[9] O. Fernández, Á. Gómez and A. Vegas, "Chiral Metamaterials with High Values of Circular Dichroism and Figure of Merit", 10th Iberian Meeting on Computational Electromagnetics (X EIEC), Baeza, Spain, May 6-8, 2015.

[10] C. Menzel, C. Rockstuhl, F. Lederer, "Advanced Jones Calculus for the classification of periodic metamaterials", Physical Review A, Vol. 82, No. 5, 053811 , Nov. 2010.

[11] Warren L. Stutzman, "Polarization in Electromagnetic Systems", Artech House, 1992. 